\title{
Exercise prescription: obeying fundamental laws of physics
}

\author{
Philip Glasgow, ${ }^{1}$ Christopher M Bleakley ${ }^{2}$
}

\section{PHYSIOS IN SPORT UK: 2012 AND BEYOND}

The year 2012 was truly special for even the most reluctant of sports fans. For many members of the Association of Chartered Physiotherapists in Sports Medicine (ACPSM), the clinical interest group representing over 1600 UK physiotherapists working in sport, 2012 was the culmination of many years of hard work and provided a unique opportunity to experience a home Olympic Games. The future looks just as exciting. January 2013 marks the start of what promises to be a fruitful affiliation between ACPSM and BJSM. Another major sporting event also edges closer, with the Commonwealth Games set to take place in Glasgow in 2014. In the lead up to this, ACPSM are holding their biennial conference in Glasgow from 25 to 26 October 2013.

A key theme of the ACPSM conference in October 2013 is to tease out the intricate relationship between anatomical form and sporting function and relate it to our capacity as clinicians to influence adaptation. Perhaps the most important modality at our disposal in this context is exercise. Our ability to prescribe the optimal type, intensity and duration of exercise can significantly influence the quality of clinical outcome. In sport and exercise medicine (SEM) it is easy to get side-tracked; magic tape, fancy injections and new age treatments can sometimes take our eye off the fundamentals. In other areas of science there are no gurus, no magic tricks and our reality is governed by the natural laws of the universe. Should SEM be any different?

The laws of thermodynamics are among the most universal and embracing in all of science; they cumulatively govern how and

\footnotetext{
${ }^{1}$ Sports Institute Northern Ireland, University of Ulster, Jordanstown, Newtownabbey, UK;

${ }^{2}$ Ulster Sports Academy, University of Ulster, Jordanstown, Newtownabbey, UK

Correspondence to Dr Philip Glasgow, Sports Institute Northern Ireland, University of Ulster, Jordanstown, Newtownabbey, BT37 OQB UK: philipglasgow@sini.co.uk
}

why energy is transferred. In the past, our physics colleagues have described the laws of thermodynamics in very simple terms, living in the universe means that: you cannot win; you cannot break even and eventually you will lose the game. The consequences of the second law of thermodynamics are perhaps the hardest hittingeventually everything in a closed system progresses from order (or low entropy) to a disordered state (or high entropy). A little depressing...

\section{INFLUENCING ADAPTATION IN DYNAMIC SYSTEMS}

However, all is not lost. As practitioners, we are not dealing with a closed deterministic system; rather we are dealing with people, living organisms consisting of cells, which allow both mass and energy to cross their membranes, all under the intricate control of functional genetic instructions ${ }^{1}$. The ability of human beings to adapt relies on the capacity of cells to transform energy into work. This is sensitive to the initial constraints of the system (individual) and will decrease with time; a concept often evidenced in longitudinal health data. A large prospective study by Sui et al found that women's fitness declined over a 7-year period, exemplified by increased body mass index and deterioration of cardiovascular fitness (see page 77).

Disease and injury are generally associated with an increase in entropy. For example, tendinopathies are characterised by histological disorder such as a loss of fibre alignment. Wider access to imaging techniques such as bedside ultrasound can help to quantify this. Comin et al reported that some sonographic tendon abnormalities were predictive of clinical symptoms in ballet dancers followed over a 24-month period (see page 89). A key concept is that in living, dynamic system it may be possible to reverse disorder. This underpins Cook and Purdam's ${ }^{2}$ recent model of tendinopathy whereby tendon health can move in either direction along a pathological continuum. A consequence is that a single image cannot distinguish if tendon changes are pathological or adaptive, or whether it is moving from low to high entropy or vice versa.

\section{HOW CAN WE PUT ENERGY INTO THE SYSTEM?}

Treatment interventions in SEM focus on the introduction or reintroduction of energy on the body. As clinicians we have a gamut of energy forms at our disposal. Manual therapy, shockwave, therapeutic ultrasound or even compression stockings can be broadly categorised as methods of delivering mechanical energy on a healing system. Haas et al have provided further evidence of the dose dependent effect associated with mechanical loading, this time in the form of compressive massage using an injured animal model (see page 83).

The optimal load or dose of energy is case specific. Mechanical loading, based on exercise and movement seems to have a special affinity with the body and may represent the most potent and effective method to present energy to a healing system.

In contrast, inactivity or the absence of mechanical loading is a powerful driver for negative-health outcomes. Further prospective analytic research can help to highlight population subgroups at most risk of sedentary behaviour (see page 71). Medicine is not perfect, and fatalistically, all living things will eventually succumb to the laws of the universe. However, according to the laws of physics, it seems exercise may be as good as it gets.

\section{Competing interests None.}

Provenance and peer review Commissioned; internally peer reviewed.

To cite Glasgow P, Bleakley CM. Br J Sports Med 2013, 47, 63.

Received 5 November 2012

Revised 5 November 2012

Accepted 5 November 2012

Br J Sports Med 2013;47:63.

doi:10.1136/bjsports-2012-091959

\section{REFERENCES}

1 Trevors JT, Saier MH Jr. Thermodynamic perspectives on genetic instructions, the laws of biology and diseased states. C R Biol 2011;334:1-5.

2 Cook JL, Purdam CR. Is tendon pathology a continuum? A pathology model to explain the clinical presentation of load-induced tendinopathy. $\mathrm{Br} / \mathrm{Sports}$ Med 2009;43:409-16. 